\title{
Research on Automatic Test of Rulestream Function Based on IBM RFT
}

\author{
JIANG Jian* \\ Teaching Affairs Department \\ Suzhou Chien-Shiung Institute of Technology \\ Taicang, China \\ E-mail: 1015671421@qq.com \\ * Corresponding Author \\ SHI Xiaolong \\ School of Mechanical Engineering \\ Yancheng Institute of Technology \\ Yancheng, China \\ E-mail: 381268703@qq.com
}

\author{
QI Wenlu \\ School of Mechanical Engineering \\ Yancheng Institute of Industry Technology \\ Yancheng, China \\ E-mail: 1015671421@qq.com
}

\author{
CHEN Canglong \\ School of Mechanical Engineering \\ Yancheng Institute of Technology \\ Yancheng, China \\ E-mail: 1542062518@qq.com
}

\begin{abstract}
How to improve the efficiency of software testing to ensure software quality and thus shorten the period of the software development, has become a very urgent task needing to handle. On the basis of the study of automated testing technology, and combined with the existed software system, this paper employs Rational Functional Tester (RFT) as the automatic software test platform. Application results show the automatic software test technique proposed in this paper greatly reduce the test cost, increase the test efficiency, and shorten the software development period obviously.
\end{abstract}

Keywords-Software Test; Testing Efficiency; Automated Testing; Testing System; RFT

\section{INTRODUCTION}

As the guarantee of providing high quality software in a limited time, the software testing is a very important part of a complete, formal software development process. With the rapid development of software technology, the efficiency of software development has been greatly improved. However, the workload of software testing has not been reduced, and software automated testing technology make the efficiency of software testing got the unprecedented ascension[1-2].

Automated testing as a software testing technology, is the process of replacing the human testing according to the plan, which is made by the test engineer through the test automation tools, and it is helpful to reduce the workload of manual testing and enhance the efficiency of the test[3]. In the process of software product development, the workload of software testing is the biggest, which can account for more than half of the entire effort. The vast majority of software testing is not the original test, which has the characteristics of high repeatability. Besides, this part is best for software automated testing to finish. In the testing process, automatic test technology can also be done to complete the test that manual test is difficult to finish[4].
In order to ensure a satisfactory testing coverage and quality of software, automated testing has become an indispensable part of the guarantee of software quality [5].

\section{THE CURRENT RESEARCH STATUS OF RELEVANT TECHNOLOGY}

With the rapid development of science and technology in China, the exchange between the foreign counterparts is increasing, and the field of software testing is becoming more and more extensive. The following will introduce the research status of domestic and foreign automated testing technology.

\section{A. Foreign research status}

Load Runner software is a very popular test tool which is developed by the foreign countries. It is mainly used in the pressure testing, performance testing and so on[6]. QTP is an automated testing tools developed by mercury company, and it is applicable to the case of a large number of redundant work. This tool has a very strong advantage in the use of drag and drop method to create test steps[7]. RFT is launched by IBM for graphical user interface. And it is very suitable for automated testing of GUI interface. IEEE and other organizations are also active in the study of the theory of software testing, and put forward a lot of new results of software testing.

\section{B. Domestic research status}

$\mathrm{S}$. Du, is a professor of Zhejiang University, puts forward the relation diagram of the interface component. Professor Liu Chao of Beihang University proposed a Program Interactive Executive Flow Chat (PIEF), etc[8]. $\mathrm{H} 3 \mathrm{C}$ has incorporated automated testing into the daily work, HUAWEI, ZTE has launched its own automated testing. Y. Lu developed a GUI automated testing system based on event flow. The GUI automated testing model is 
useful and provides a more effective way to improve the automation of GUI testing in Windows environment.

\section{REALIZATION OF AUTOMATED TESTING S YSTEM}

\section{A. Automated testing scripts}

\section{1) Testing script writing}

Click the start button recording, and then input the name of the script. The RFT script recording is shown as Fig. 1.

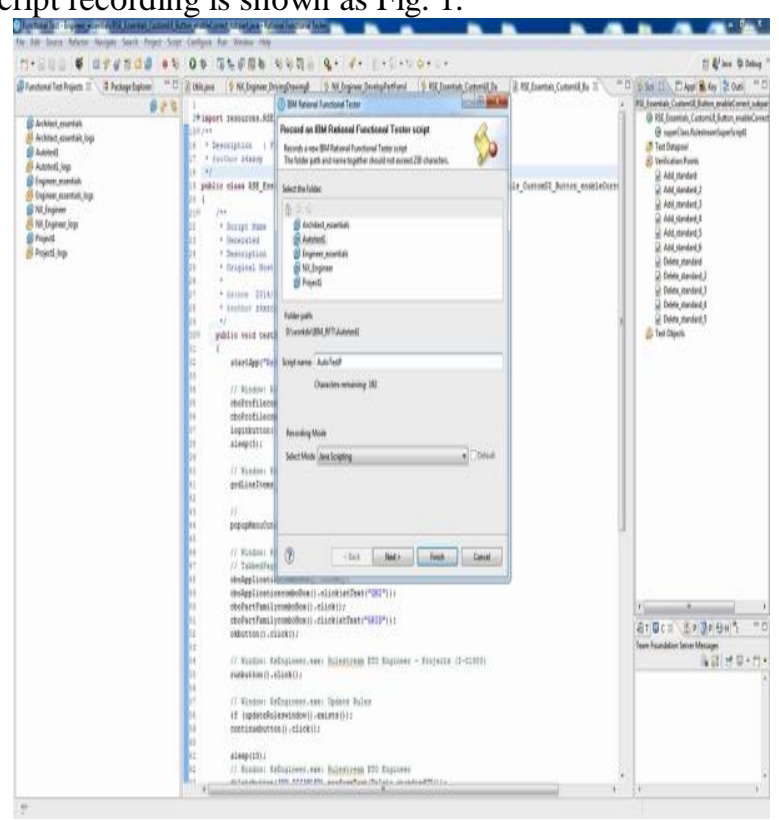

Figure 1. RFT script recording

The database format of this script is "rfdtp ."Click Finish to enter the recording mode.

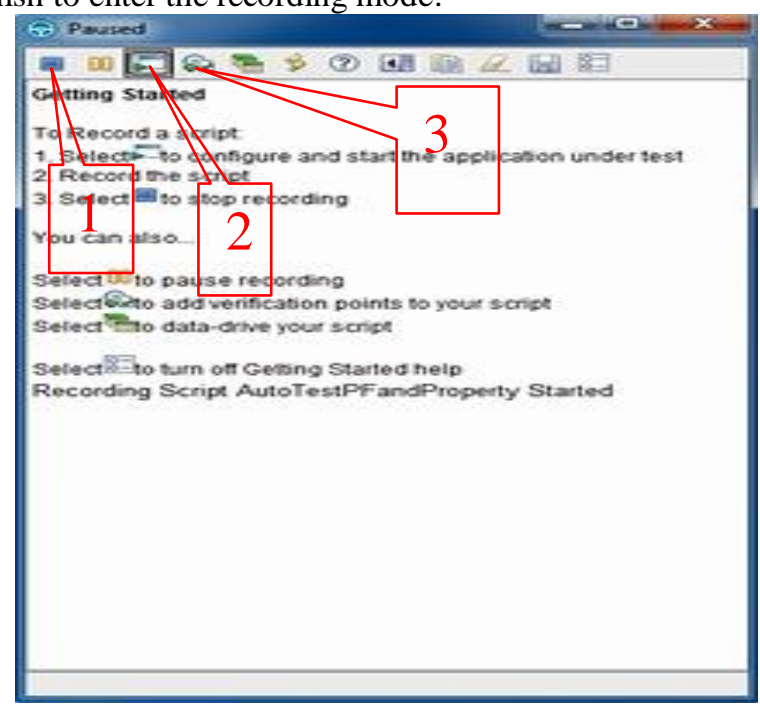

Figure 2. RFT toolbar

In Figure 2, Label 1 means the End Button, Label 2 the Program Start Button, and Label 3 the Key Point Testing Button. Each operation will generate the corresponding code stored in the Script. researchers can also have a special test to some key points. The test of key point is shown as Fig. 3.

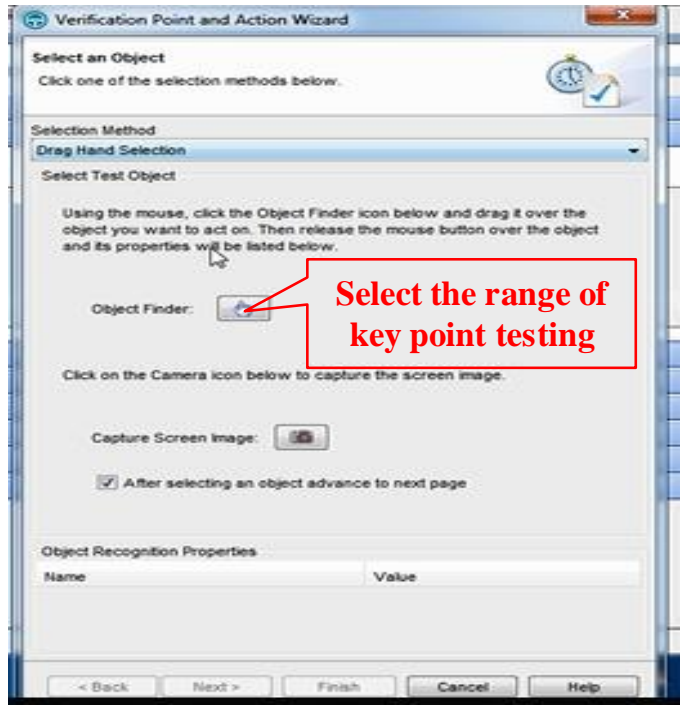

Figure 3. Key point testing

Finally, click End button, it will form a complete set of test scripts. However, there are a lot of redundant parts of the test program, and researchers need to analyze the program to remove the excess part, so that the process is more refined.

2) Script analysis

Tester judge the procedural steps is in line with the requirements according to the generated scripts. It will also add some special procedure steps to make the automated testing framework more standardized.

Here is a section of the script, as an example of the analysis of the part of the program:

tartApp("RsArchitect");

Start Rulestream software

sleep (2);

Sleep 2 seconds

cboProfilecomboBox().click();

Click cboProfilecomboBox dialog box

cboProfilecomboBox().click(atText("Architect_essenti als_RFT"));

Select the Architect_essentials_RFT in the cboProfilecomboBox

loginbutton().click();

Login Software

rulestreamETOArchitectwindow().maximize();

Maximize software

toolStripArchitecttoolBar().click(atText("New Part

Family"),

Input the name of member family part 1

toolStripArchitecttoolBar().click(atButton(atText("Sav e")));

Click the save button

ultraTabControlltabbedPage().click(CLOSE_BUTTO $\mathrm{N})$;

Click the close button

tvwPartFamily_Ultratree().click(atPath("part1->Locati on(CAPTION)"));

Select the caption in part1

tvwPartFamily_Ultratree().click(RIGHT, atPath("part1->Location(CAPTION)"));

Right click caption contextMenuStriptoolBar().click(atPath("Delete"));

Click the delete button in the toolbar 
getScreen().inputKeys(" $\{\mathrm{TAB}\} ")$;

Press TAB button

getScreen().inputKeys("\{ENTER $\} ")$;

Press ENTER button

txtSystemNametext().performTest(txtSystemName_tex $\operatorname{tVP}())$

Special checkpoint procedures

getScreen().inputChars("D: \\Rulestream Idocument \D

atabasesand Docs $\backslash$ MasterDocs $\backslash$ MasterDocs\\Block.jpg");

Input the path, open the Block.jpg file

if (!SingleView_Checked)

singleViewcheckBox().clickToState(SELECTED);

Conditional statements, if SingleView is not selected, then click the select button

$\operatorname{rtftext}() \cdot$ setText("result $=$ me.Length $*$ me.Width $*$

me.Height");

Input the formula of length * width * height

ultraTabControlltabbedPage().click(RIGHT,

atPosition $(5,10))$;

Right-click the coordinates of $(5,10)$

contextMenuStriptoolBar().click(atPath("Check In"));

Click the move commandin the toolbar;

rulestreamETOArchitectwindow(ANY,MAY_EXIT).c

lick(CLOSE_BUTTON);

Click Close eject button in the toolbar;

\section{B. Automated testing principle of RFT}

\section{1) Principle of code}

After the launch of the RFT script recording, the user can make a series of operations on the target page, which will be recorded and stored in an object mapping table ${ }^{[8]}$. This object mapping table is a tree structure table, and each recorded object is placed among them according to its position in the page component structure. When running RFT for automated testing, RFT calls the object map of the information on the server to carry out the relevant operations. Choose the Script $=>$ Open the Test Object Map in the RFT toolbar to open the object mapping table. The object mapping table is shown as Fig. 4.

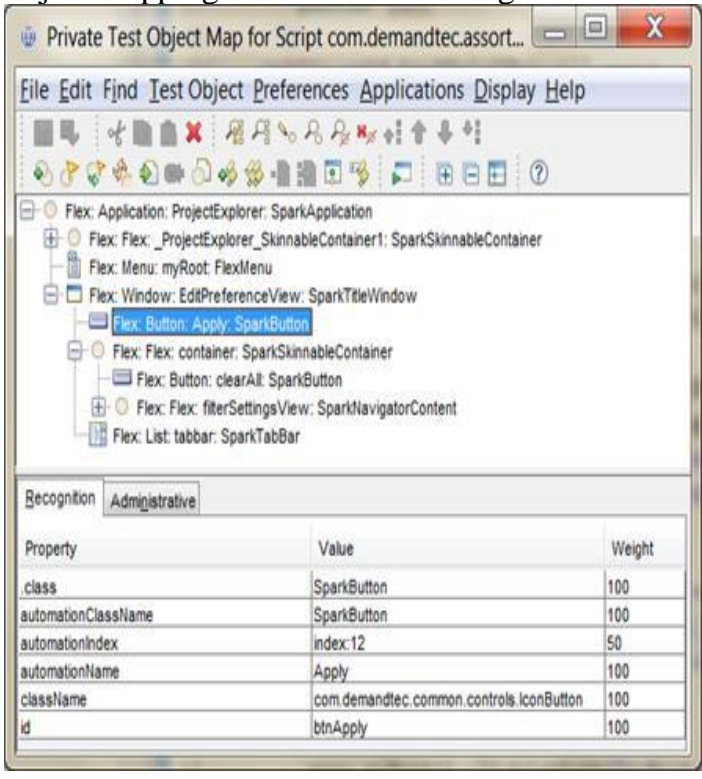

Figure 4. Object mapping table

\section{2) Recognition process}

A brief description of the process of object recognition in the test program is described here. If researchers need to click on the highlight object represented by the button in the testing process, RFT first needs to find the GUI object represented by the root of the object tree. It is not hard to see that the object on the root of the tree represents a Java of the Frame. As long as the correct Frame is launched, the object on the root is not hard to find. RFT will keep looking step by step according to the tree structure until find the corresponding button, so that researchers can execute the button click operation in the program. Thus, it is important to identify all the objects in the tree. As long as one of them can not be identified, then the button will not be found in the end. And RFT will determine whether the object is in accordance with the criterion of identification according to an algorithm in the process of identifying the GUI object[9].

The common automated test development process of Rulstream based on RFT is to record the operations using RFT on the local computer, then modify and debug the generated code, finally realize automated testing on the local computer. The case cannot be run on the server, because that RFT function cannot identify Rulestream internal menu, such as right click menu and data sheets, etc., which leads to the way of data recording is through coordinate record, rather than menu attributes.

In daily application, it is inevitable to meet the secondary window of Windows, such as dialog saving hint, dialog deleting hint, etc. Rulestream is no exception. However, in Rulestream-based automatic test system, RFT cannot recognize secondary windows of Windows operation system, which causes the operation record of secondary windows of Windows operation system is blank.

\section{TEST CASE}

The case used in this paper check whether NX will follow the new changes when tester changes the parameters of Rulestream. A cans model is used as a case.

\section{A. Process of automated testing}

1) Carry out related operations in RSA;

a) Add the related PartyFamily

b) Add Spec NX and add BeerCan.prt

c) Set up checkpoints to detect whether BeerCan.prt added successfully

d) Bind related properties

e) Set up checkpoints to detect whether properties of Rulestream added and bound successfully

2) Carry out related operations in RSE;

a) Build and perform new Project and LineItem

b) Modify RS Property

c) Release Model

3) Write Code for RFT.

a) Set waiting time

b) Modify the code generated by wrong operation

c) Detect checkpoints

d) Add detection of NX Part 


\section{B. The implementation of automated testing}

1) Open the RFT software. Configure the required parameters and make sure that start button of the RFT can complete the start of the Rulestream; The setting window of RFT parameter is shown as Fig. 5.

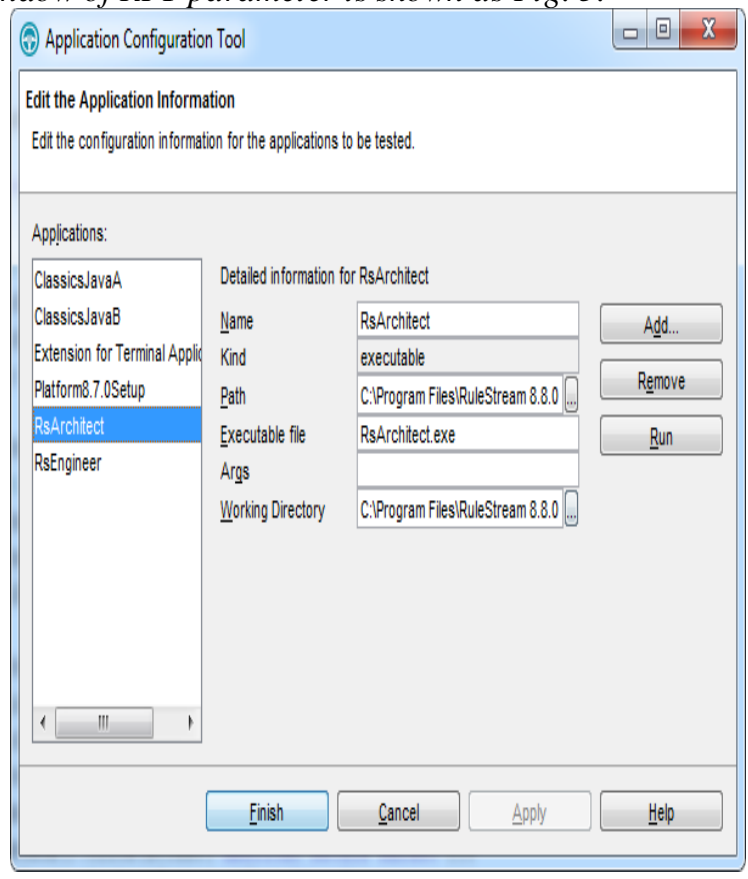

Figure 5. The setting window

2) Click the record button to start recording. The recording window is shown as Fig. 6.

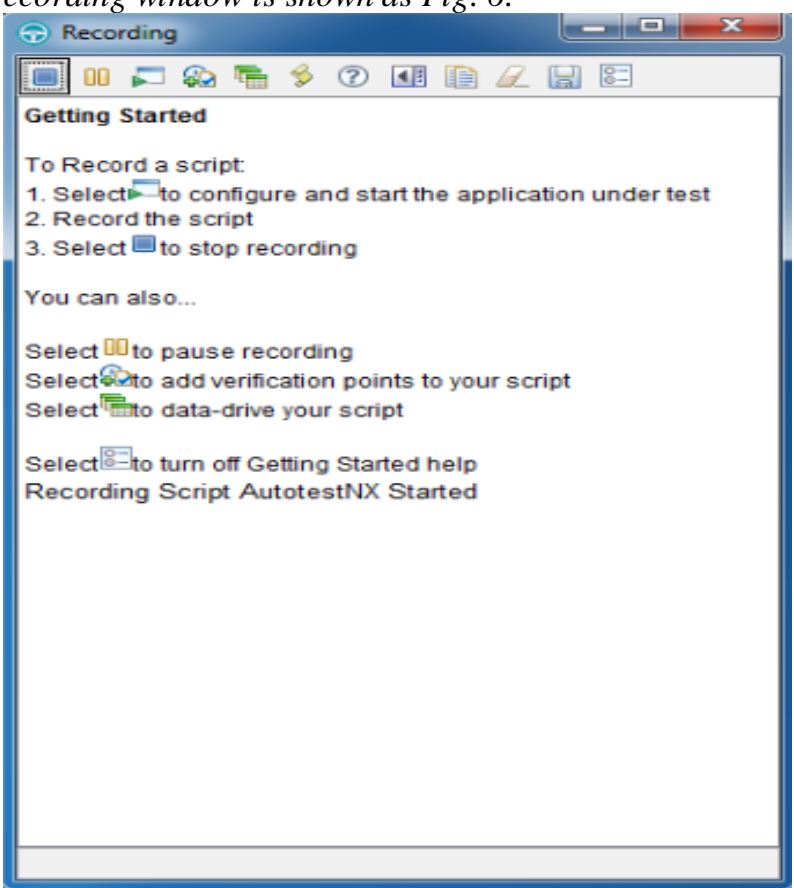

Figure 6. The recording window

3) Start RSA, and then carry out the related operation to SRA, add Part Family; The window of NX Spec is shown as Fig. 7.

4) Add NX Spec, and bind the properties of Rulestream;
5) Adding detection point, detect the above steps to success;

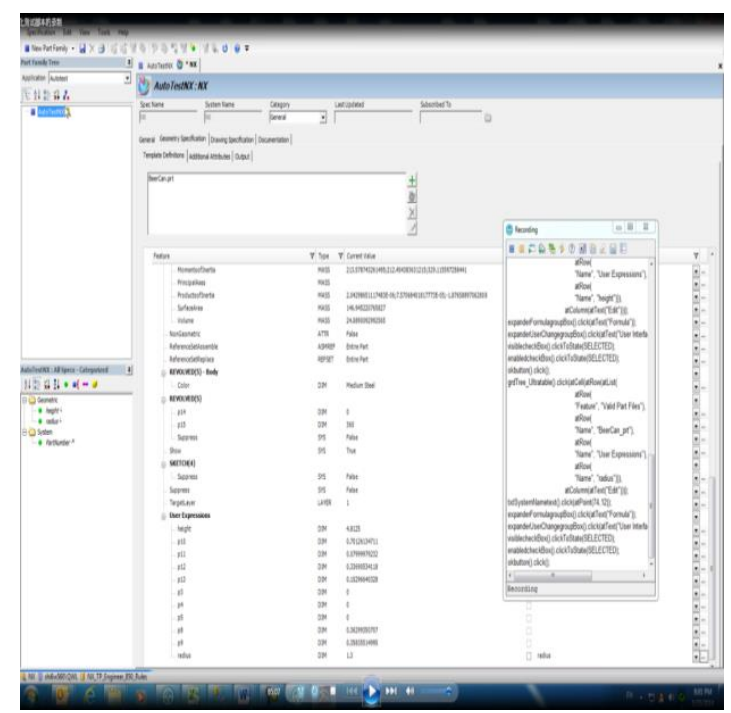

Figure 7. The window of NX Spec

6) Complete the RSA test, start the RSE recording, create and execute new Project and Line Item the recording interface is shown as Fig. 8.

7) End RSE recording, complete the entire recording of automated script.

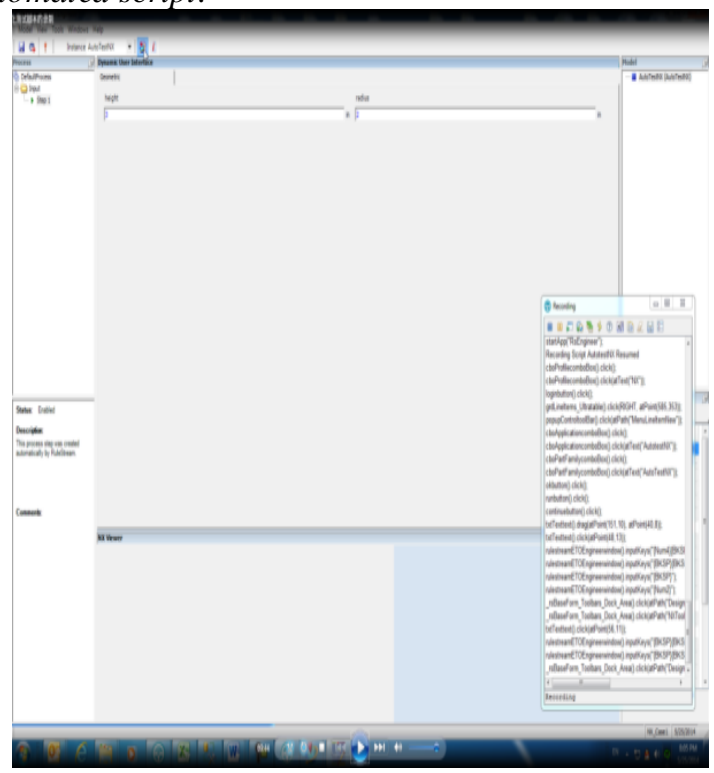

Figure 8. RSE recording interface

C. Modify the code generated by RFT

1) In order to ensure that the server has a full time to response at run time, tester can add sleep (10) after Loginbutton ().Click (), etc. Examples are as follows:

cboProfilecomboBox(). $\operatorname{click}()$;

cboProfilecomboBox().click(atText("NX"));

loginbutton().click();

// Window: RsArchitect.exe: Rulestream ETO Architect

toolStripArchitecttoolBar().click(atText("New Part

Family"), 
Tester can change it into the following program. cboProfilecomboBox().click(); cboProfilecomboBox().click(atText("NX")); loginbutton().click(); sleep (5); // Window: RsArchitect.exe: Rulestream ETO Architect toolStripArchitecttoolBar().click(atText("New Part Family"),

2) Delete some code generated by wrong operation comboBoxcomboBox().click(atPoint $(95,13)$ ); comboBoxcomboBox().click(atPoint $(110,12))$; openFilewindow().inputKeys("E:Ilworkdirl|QAllModel

s\\L_NXI|BeerCan.par\{BKSP $\}\{$ BKSP $\} r t ")$; openFilewindow().inputKeys(" $\{$ ENTER $\} ")$; Tester can change it into the following program. comboBoxcomboBox().click(atPoint $(95,13)$ ); $/ /$ comboBoxcomboBox().click(atPoint $(110,12))$; openFilewindow().inputKeys("E: Ilworkdir|\QA \Model s\\L_NX\\BeerCan.prt"); openFilewindow().inputKeys("\{ENTER\}");

// Window: RsArchitect.exe: Add File _Importbutton().click();

3) Modify the code can not run nobutton(ANY,MAY_EXIT).click();

Tester can change it into the following program. getScreen().inputKeys("\{TAB $\} ")$; getScreen().inputKeys("\{ENTER $\} ")$;

4) Add the code, extract information from the NX model generated by Release, generate XML file, and compare the XML file.

Utils util = new Utils () ;

util.validateResult(

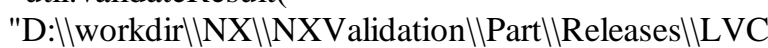
000005.0001.00 01॥RefSystem-(LVC000005-1-17).prt",

"NX_Engineer_DevelopPartFamilyTreeInRSE1");

Due to the complexity of the process of script recording, the generated or modified code may not replay successfully. So the final automated testing scripts need several debugging.

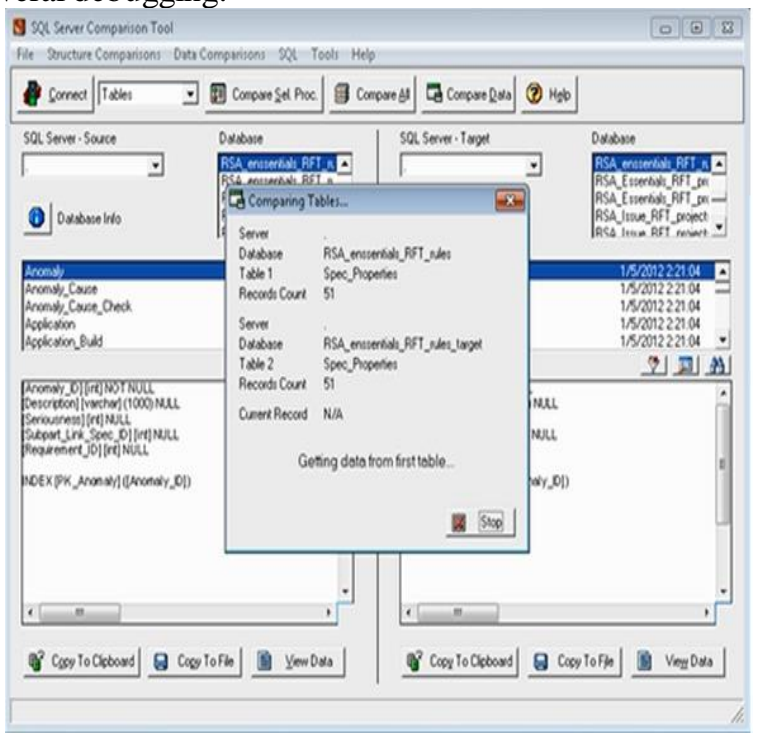

Figure 9. Data comparison window
When the new version of the software is released, researchers will play back the recorded operation in order to check the software features have no degradation phenomenon. The basis of the examination is to compare the playback data with the data generated from the correct operation. The tester can analysis and study whether there is something wrong with the function of the software. The data comparison window is shown as Fig. 9.

\section{CONCLUSION}

The design and implementation of automatic test system based on RFT is introduced in this paper. Rational Function Tester, enterprise automation software, is used in this system to test Rulestream which is industry software of Siemens. This paper designs and implements a graphical user interface of the automated test scripts combined with the RFT on the base of the recent research of the key technology of GUI testing. The automatic test can reach the same effect of the manual test through debugging. As long as IBM RFT is used for the automatic detection of the function of industry software Rulestream, it can greatly reduce the test cost, increase the test efficiency, and can shorten the software development cycle.

In spite of the automated testing based on RFT has had a good application in specific projects, there are also many place need to improve such as the reuse of test scripts and the optimization of automated test case.

\section{ACKNOWLEDGMENT}

The authors gratefully acknowledge the support by the Jiangsu Province Modern Education Technology Research Project Under Grant No. 2014-R-33872.

\section{REFERENCE}

[1] D. Lu, "Application and Research of Testing Automation in Large Scale System," Zhejiang: Zhejiang University, 2010.

[2] Y. Jiang and J. Zhao, "An Approach to Automatic Generation of Test Automation Scripts," Computer Technology and Development, no. 07, 2007, pp.4-7.

[3] Y. Cao, "The design and application of WEB test automation framework based on RFT," Liaoning: Dalian University of Technology, 2013.

[4] J. Tao, "The Platform of Automate Test System Design and Implementation," Sichuan: University of Electronic Science and Technology of China, 2013.

[5] J. J. Xie, "The Design and Implementation of RFT-based Web System Automation Testing," Jiangsu: Nanjing University, 2012.

[6] C. Carlson and L. V. Kirkland, "The test executive: the strength of testing and diagnosis," IEEE Transactions on Computers, vol. 12, no. 3, 2008, pp.175-180

[7] E. Larsson and S. Edbom, "Test data truncation for test quality maximization under ATEmemory depth constraint," IET Computers \& Digital Techniques, vol.1, no.1, 2007, pp.26-38.

[8] F. Zhang, "Design and Implementation of Automated Testingof WEB Interface Based on the RFT Software," Hubei: Huazhong University Of Science And Technology, 2013.

[9] M. Xi and F. Zhao, "Data-model-driven Software Automation Test Framework," Computer Engineering, no.21, 2009, pp.78-81.

[10] L. Cong, "The application of IT in auality management of construction project," WMWA, 2010. 